\title{
LAS CONSECUENCIAS ECONÓMICAS DEL CORONAVIRUS ${ }^{\circ}$
}

\author{
THE ECONOMIC CONSEQUENCES OF CORONAVIRUS
}

\author{
Marcos Vasconcelos*
}

enviado: 15 abril 2020 - aceptado: 27 abril 2020

\begin{abstract}
Resumen
Esta nota presenta reflexiones sobre los posibles impactos que la pandemia de coronavirus tendrá en la economía. La interrupción de las cadenas de producción, la caída de la producción y los ingresos son impactos inmediatos. Sin embargo, también se espera que los efectos a largo plazo sean significativos y negativos para el crecimiento económico futuro.

Palabras clave: pandemia de coronavirus, COVID-19, crisis económica, crecimiento económico, deuda pública.

Código JEL: E02, E60, F01.
\end{abstract}

\begin{abstract}
This note presents reflections on the potential impacts that the coronavirus pandemic will have on the economy. The interruption of production chains, the fall in production and income are immediate impacts. However, the long-term effects are also expected to be significant and negative for future economic growth.

JEL codes: E02, E60, F01

Keywords: Coronavirus pandemic, COVID-19, economic crisis, economic growth, public aid.

Vasconcelos M. (2020). Las consecuencias económicas del Coronavirus. Estudios económicos, 37 (75), 131-138.

* Universidade Estadual de Maringá (UEM), Departamento de Ciencias Económicas. Correo electrónico: mrvasconcelos@uem.br
\end{abstract}





\section{INTRODUCCIÓN}

No es exagerado decir que la economía mundial se somete a una de sus mayores pruebas. La nueva pandemia de coronavirus (SARS-CoV-2) parece ser uno de esos eventos raros en la historia, pero cuyos impactos tienden a ser significativos, aunque inciertos, a corto, mediano y largo plazo. Usando el concepto de Taleb (2010), el mundo sufre los efectos de un evento de "cisne negro".

Existe un amplio esfuerzo de los más diversos campos de la ciencia para producir conocimiento que ayude a la humanidad a superar el momento. En primera línea, las ciencias de la salud buscan frenéticamente formas de comprender y enfrentar la enfermedad COVID-19.

La economía, por otro lado, ha tratado de ayudar a formular acciones y mecanismos no farmacológicos capaces de mitigar los efectos sobre los ingresos y las actividades económicas, pero también previendo el período posterior a la pandemia. El hecho de que la velocidad e intensidad de la pandemia haya envejecido todos los escenarios económicos asumidos anteriormente hace que esta misión sea más desafiante. El nuevo coronavirus ha producido ondas de choque de incertidumbre en el entorno económico. A pesar de este contexto, algunas consideraciones son posibles.

\section{DESAJUSTE ENTRE LA OFERTA Y LA DEMANDA}

Se ha demostrado que la alta tasa de diseminación del nuevo coronavirus y los efectos de COVID-19 en la salud pública pueden impulsar, en poco tiempo, una demanda de atención médica mucho más allá de la capacidad de suministro médico presente en la mayoría de los países. Tal situación, sumada a la falta de un arsenal farmacológico adecuado para combatir los casos más graves de COVID-19, rápidamente demostró ser capaz de inducir el colapso de los sistemas de salud y amenazar la vida de miles de personas.

Sin entrar en el campo de las enfermedades infecciosas, ya debería estar claro para los economistas que el desequilibrio entre la demanda y la oferta de servicios de atención médica ha demostrado ser un factor que influye en la tasa de mortalidad de la enfermedad en diferentes lugares. Por lo tanto, el esfuerzo para evitar el colapso del sistema hospitalario se vuelve urgente. Como la capacidad de ajustar el lado de la oferta, especialmente en el caso de una pandemia global, es limitada, queda para la sociedad y sus gobiernos tratar de manejar el lado de la demanda. 
En este contexto, ha surgido el principio de prudencia. Varios países y localidades han comenzado a imponer a su población la única opción disponible por el momento para evitar que el impacto de COVID-19 sea catastrófico: la adopción de la llamada distancia social (Ferguson et al., 2020). Aunque se enfrenta a un escenario lleno de incertidumbre en el que el principio de precaución recomienda que la distancia social sea lo más amplia y profunda posible, no todos los gobiernos nacionales o locales la siguieron de inmediato.

Sin embargo, el triste desarrollo de los hechos se ha impuesto a los intereses ideológicos de los líderes nacionales, casos, por ejemplo, de los Estados Unidos e Inglaterra, o en una concepción imaginaria de "nuestra sociedad es diferente", como en Suecia. Cuatro meses después del comienzo de la crisis del coronavirus, actualmente pocos gobiernos nacionales continúan negando o minimizando la gravedad de la situación causada por COVID-19 en la salud pública.

\section{FALSO DILEMA ENTRE ECONOMÍA O SALUD}

En situaciones más graves, como Italia y España, o en las que los líderes políticos fueron más firmes en la lucha contra la amenaza de la pandemia, como en Nueva Zelanda, el desapego social ha tomado la forma de aislamiento social, poniendo a la mayoría de la población en cuarentena. Ya sea por distanciamiento o aislamiento social, el esfuerzo por preservar vidas ha sido el corolario de interrumpir varias actividades económicas. Incluso las actividades consideradas esenciales se han visto afectadas. Y aunque la cadena de producción no se ve directamente afectada por la pandemia, la parálisis del comercio y, en consecuencia, de las ventas transmite la crisis a la producción. Y la conmoción de incertidumbre producida por la pandemia ya deja una recesión económica contraída para los próximos trimestres. La crisis de salud va acompañada de una debacle económica.

Es importante tener en cuenta que las experiencias actuales e históricas muestran que no hay una compensación entre las dos crisis, es decir, el impacto económico de la pandemia no puede reducirse al aceptar una propagación más rápida del coronavirus. Por el contrario, como demostraron Correia, Luck y Verner (2020), hay evidencia de que, durante la pandemia de gripe española, en 1918, las ciudades de América del Norte que actuaron con más rigor en la lucha contra las enfermedades y la contaminación mostraron menos daños económicos durante la pandemia y una recuperación más sólida después. 


\section{ARENA EN LOS ENGRANAJES DE LA ECONOMÍA GLOBAL}

A nivel internacional, los efectos están empeorando. Varias cadenas de suministro se ven afectadas y los engranajes de la economía mundial dejan de funcionar correctamente. La caída del comercio internacional se ha acentuado y, de esta manera, el espectro de la parálisis económica se está extendiendo por toda la economía mundial y proyectando sombras para el período posterior a la pandemia.

La recuperación del crecimiento económico será más difícil en un escenario de menor comercio internacional y con la adopción de restricciones sanitarias que obligarán a varios cambios en la asignación de recursos, muchos de ellos no siempre hacia una mayor eficiencia. Todas las actividades económicas que dependen de alguna forma de aproximación social sufrirán para volver al nivel anterior a la pandemia. Probablemente habrá que revisar varios e importantes procesos de producción con la búsqueda de proveedores locales. Y el movimiento para reducir continuamente los costos de producción industrial podría detenerse.

\section{LA ACCIÓN IMPERATIVA Y URGENTE DEL ESTADO}

En el corto plazo, la distancia social necesaria ha estado afectando las vidas de miles de personas cuya fuente de ingresos estaba de alguna manera vinculada al movimiento de personas y la cercanía social. En países, como los de América del Sur, en los que las relaciones económicas, especialmente las de trabajo, a menudo carecen de una red de seguridad social, la interrupción de varias actividades implica la pérdida inmediata de empleos e ingresos. El colapso de la economía puede convertirse en un colapso social si no se remedia rápidamente mediante acciones estatales.

El seguro de desempleo y los sistemas de suplementación de ingresos deben implementarse de manera urgente. En la medida de lo posible, deben ser focalizados y reforzados especialmente para segmentos de la población con mayor vulnerabilidad socioeconómica. La interrupción de las actividades económicas puede incluso afectar a todos por igual, pero sus consecuencias son mucho más graves para aquellos con relaciones laborales informales y que realizan actividades intermitentes, es decir, la parte más pobre de la población. La desigualdad de ingresos existente tiende a profundizarse sin la acción del Estado.

La necesidad de que los estabilizadores de ingresos tomen medidas aumenta la expansión del gasto en salud y ambos presionan el gasto público. Al mismo tiempo, la parálisis de las actividades económicas reduce los ingresos estatales en 
diferentes niveles de gobierno. El deterioro fiscal debe tratarse como normal en una situación excepcional. Y parte del costo del ajuste debe pasar a las generaciones futuras en forma de una mayor deuda pública.

\section{LAS CONSECUENCIAS ECONÓMICAS DE LA PANDEMIA}

La experiencia de las décadas posteriores a la Segunda Guerra Mundial nos impide decir que el aumento de la deuda pública necesariamente tendrá como resultado un menor crecimiento económico a largo plazo (Abbas et al., 2011). Varios países tenían altos niveles de deuda pública en 1945 y esto no impidió la recuperación económica global observada en la secuencia. Sin embargo, existe una diferencia significativa entre la anormalidad económica generada por el conflicto mundial de la década de 1940 y la crisis actual. Esa enorme destrucción del stock de capital ocurrió en algunas de las economías más importantes del mundo y, después del conflicto, hubo oleadas de gasto de inversión que ayudaron a la recuperación de la economía global. Este no es el caso con la crisis actual. Por el contrario, la tendencia es que la inversión caiga.

La interrupción de las actividades económicas dejará consecuencias. Por el lado de la oferta, las empresas terminarán esta crisis con pocos recursos en efectivo, obligándolos a revisar los proyectos de inversión. Otros, debilitados financieramente, pero con buenos activos, serán objetivos para el proceso de concentración del mercado que debería ocurrir en varios sectores de la economía. Los más frágiles desaparecerán. Por el lado de la demanda, la tendencia es aumentar el endeudamiento promedio y el empobrecimiento de las familias.

Por lo tanto, la combinación de ajustes de oferta y demanda será la normalización de la demanda agregada a un nivel y a un ritmo más suave que el observado en la fase que precede a la pandemia. La propia confianza de los agentes económicos se recuperará solo lentamente. Estas tienden a ser las implicaciones a medio plazo de la crisis.

\section{NUBES EN EL HORIZONTE}

Es más difícil predecir los impactos en las tendencias económicas a largo plazo. Después de todo, dependen de las soluciones que la ciencia de la salud podrá desarrollar para tratar con COVID-19. Con las drogas o vacunas existentes capaces de llevar la tasa de mortalidad de esta enfermedad a niveles insignificantes, las rela- 
ciones económicas internacionales tienden a normalizarse, ya sea en el comercio o el turismo. De lo contrario, tales actividades, entre otras, se restablecerán en niveles inferiores a los observados en las últimas décadas.

Incluso en el mejor de los casos, las cadenas de producción mundiales pueden sufrir reajustes que amplíen, cuando sea posible, la presencia de proveedores locales, incluso si los grandes conglomerados aumentan la seguridad de sus líneas de producción con proveedores redundantes. Los sectores de comercio, turismo, entretenimiento y educación, por ejemplo, pueden verse afectados por el uso acelerado de nuevas tecnologías. En el mundo del trabajo, debe profundizarse el uso de tecnologías capaces de reemplazar las actividades humanas.

\section{CONCLUSIONES}

En resumen, además de su grave impacto en la salud pública, la COVID19 tiene el potencial de causar cambios en las preferencias de los consumidores, interrupciones y revisiones en las estructuras de suministro, choques negativos en la productividad, brotes de incertidumbre, disminución de la confianza y destrucción de empleo en todo el mundo. Desafortunadamente, esta no es una combinación muy prometedora. Pero sus consecuencias finales dependerán de qué posiciones políticas emergerán con mayor fuerza para dictar las direcciones económicas y sociales al final del brote de coronavirus.

\section{REFERENCIAS BIBLIOGRÁFICAS}

Abbas, S. M. A., Blhocine, N., El-Ganainy, A., \& Horton, M. (2011). Historical patterns and dynamics of public debt: evidence from a new database. IMF Economic Review, 59 (4), 717-742.

Correia, S., Luck, S., \& Verner, E. (2020). Pandemics depress the economy, public health interventions do not: evidence from the 1918 Flu. Recuperado de https://ssrn.com/abstract=3561560 o http://dx.doi.org/10.2139/ssrn.3561560.

Ferguson, N., Laydon, D., Nedjati-Gilani, G., Imai, N., Ainslie, K., Baguelin, M., ..., \& Bhatia, S. (2020). Impact of non-pharmaceutical interventions (NPIs) to reduce COVID-19 mortality and healthcare demand. Recuperado de https://imperial.ac.uk/media/imperial-college/medicine/sph/ide/gida-fellowships/Imperial-College-Covid19-NPI-modelling-16-03-2020.

Taleb, N. (2010). The Black Swan: the impact of the highly improbable. New York: Random House. 
(C) 2020 por los autores; licencia no exclusiva otorgada a la revista Estudios económicos. Este artículo es de acceso abierto y distribuido bajo los términos y condiciones de una licencia Atribución-No Comercial 4.0 Internacional (CC BY-NC 4.0) de Creative Commons. Para ver una copia de esta licencia, visite http://creativecommons.org/licenses/by-nc/4.0 